\title{
LINEAR MAPS PRESERVING KY FAN NORMS AND SCHATTEN NORMS OF TENSOR PRODUCTS OF MATRICES
}

\author{
AJDA FOŠNER*, ZEJUN HUANG ${ }^{\dagger}$, CHI-KWONG LI ${ }^{\ddagger}$, AND NUNG-SING SZE ${ }^{\S}$
}

\begin{abstract}
For a positive integer $n$, let $M_{n}$ be the set of $n \times n$ complex matrices. Suppose $\|\cdot\|$ is the Ky Fan $k$-norm with $1 \leq k \leq m n$ or the Schatten $p$-norm with $1 \leq p \leq \infty(p \neq 2)$ on $M_{m n}$, where $m, n \geq 2$ are positive integers. It is shown that a linear map $\phi: M_{m n} \rightarrow M_{m n}$ satisfying

$$
\|A \otimes B\|=\|\phi(A \otimes B)\| \quad \text { for all } A \in M_{m} \text { and } B \in M_{n}
$$

if and only if there are unitary $U, V \in M_{m n}$ such that $\phi$ has the form $A \otimes B \mapsto U\left(\varphi_{1}(A) \otimes \varphi_{2}(B)\right) V$, where $\varphi_{s}(X)$ is either the identity map $X \mapsto X$ or the transposition map $X \mapsto X^{t}$. The results are extended to tensor space $M_{n_{1}} \otimes \cdots \otimes M_{n_{m}}$ of higher level. The connection of the problem to quantum information science is mentioned.
\end{abstract}

AMS subject classifications. 15A69, 15A86, 15A60, 15A18.

Key words. Complex matrix, linear preserver, spectral norm, Ky Fan $k$-norm, Schatten $p$-norm, tensor product.

1. Introduction and preliminaries. For a positive integer $n$, let $M_{n}$ be the set of $n \times n$ complex matrices. Now, suppose that $m, n \geq 2$ are positive integers. Then for $A \in M_{m}$ and $B \in M_{n}$, we denote by $A \otimes B \in M_{m n}$ their tensor product (a.k.a. the Kronecker product). In many applied and pure studies, one considers the tensor product of matrices; for example, see $[2,9,18,21]$. Most noticeably, the tensor product is often used in quantum information science [19]. In a quantum system, quantum states are represented as density matrices (positive semi-definite matrices with trace one). Suppose $A \in M_{m}$ and $B \in M_{n}$ are two quantum states in two quantum systems. Then their tensor product $A \otimes B$ describes the joint state in the bipartite system, in which the general states are density matrices in $M_{m n}$. More generally, one may consider tensor states and general states in a multipartite system $M_{n_{1}} \otimes \cdots \otimes M_{n_{m}}$ identified with $M_{N}$ where $N=\prod_{i=1}^{m} n_{i}$.

In general, it is relatively easy to construct and extract information from matrices in tensor product form. For instance, the eigenvalues (respectively, the singular values) of $A \otimes B$ have the form $a_{i} b_{j}$ with $1 \leq i \leq m$ and $1 \leq j \leq n$ if $A \in M_{m}$ and $B \in M_{n}$ have eigenvalues (respectively, singular values) $a_{1}, \ldots, a_{m}$ and $b_{1}, \ldots, b_{n}$, respectively. Thus, it is interesting to get information on the tensor space $M_{m n}$ by examining the properties of the small collection of matrices in tensor form $A \otimes B$. In particular, if we consider a linear map $\phi: M_{m n} \rightarrow M_{m n}$ and if one knows the images $\phi(A \otimes B)$ for $A \in M_{m}$ and $B \in M_{n}$, then the map $\phi$ can be completely characterized as every $C \in M_{m n}$ is a linear combination of matrices in tensor form $A \otimes B$. Nevertheless, the challenge is to use the limited information of the linear map $\phi$ on matrices in tensor form to determine the structure of $\phi$. In [5], we considered linear maps preserving the spectrum $\sigma(A \otimes B)$ and spectral

* Faculty of Management, University of Primorska, Cankarjeva 5, SI-6104 Koper, Slovenia. (Email: ajda.fosner@fm-kp.si)

$\dagger$ Department of Applied Mathematics, The Hong Kong Polytechnic University, Hung Hom, Hong Kong. (Email: huangzejun@yahoo.cn)

$\ddagger$ Department of Mathematics, College of William and Mary, Williamsburg, VA 23187, USA; Department of Mathematics, University of Hong Kong, Pokfulam, Hong Kong. (Email: ckli@math.wm.edu)

$\S$ Department of Applied Mathematics, The Hong Kong Polytechnic University, Hung Hom, Hong Kong. (Email: raymond.sze@polyu.edu.hk) 
radius $r(A \otimes B)$ of Hermitian matrices $A \in M_{m}$ and $B \in M_{n}$. In [10], the author considered linear maps $\phi: M_{m n} \rightarrow M_{m n}$ satisfying

$$
\left\|\left|A \otimes B \left\|\left|=\left\||\phi(A \otimes B) \|| \quad \text { for all } A \in M_{m} \text { and } B \in M_{n}\right.\right.\right.\right.\right.
$$

where $\||\cdot|\|$ is a certain (separability) norm defined in [11]. This family of (separability) norms was shown to be related to the problem of detecting bounded entangled non-positive partial transpose states and characterizing $k$-positive linear maps in quantum information science.

Suppose $X \in M_{N}$ has singular values $s_{1}(X) \geq \cdots \geq s_{N}(X)$. The Ky Fan k-norm of $X$ is defined by

$$
\|X\|_{(k)}=s_{1}(X)+\cdots+s_{k}(X) .
$$

The Ky Fan 1-norm reduces to the spectral norm and the Ky Fan $N$-norm is also called the trace norm. For $p \geq 1$, the Schatten $p$-norm of $X$ is defined by

$$
\|X\|_{p} \equiv\left(\sum_{i=1}^{N} s_{i}(X)^{p}\right)^{1 / p} .
$$

The limiting case $p=\infty$ is just the spectral norm, $\|\cdot\|_{1}$ is the trace norm, and $\|\cdot\|_{2}$ is the Frobenius norm, i.e., $\|X\|_{2}=\left(\operatorname{tr}\left(X X^{*}\right)\right)^{1 / 2}$. In bipartite quantum systems, a well known criterion for separability of a state is the computable cross norm (CCNR) criterion [4, 20], which asserts that if a state (density matrix) $X$ in $M_{m n}$ is separable, the trace norm of the realignment of $X$ (see e.g. [4]) is at most 1.

The purpose of this paper is to study linear maps $\phi: M_{m n} \rightarrow M_{m n}$ satisfying

$$
\|A \otimes B\|=\|\phi(A \otimes B)\| \quad \text { for all } A \in M_{m} \text { and } B \in M_{n}
$$

where $\|\cdot\|$ denotes the Ky Fan $k$-norm with $1 \leq k \leq m n$, or the Schatten $p$-norm with $1 \leq p \leq \infty$.

Note that even if we know that $\phi: M_{m n} \rightarrow M_{m n}$ is linear and satisfies $\|A \otimes B\|=\|\phi(A \otimes B)\|$ for all $A \in M_{m}$ and $B \in M_{n}$, it does not ensure that $\left\|A_{1} \otimes B_{1}+A_{2} \otimes B_{2}\right\|=\left\|\phi\left(A_{1} \otimes B_{1}\right)+\phi\left(A_{2} \otimes B_{2}\right)\right\|$ because $A_{1} \otimes B_{1}+A_{2} \otimes B_{2}$ may not be of the form $A \otimes B$. Thus, the proofs of our main results (Theorems 2.6 and 2.9) are quite delicate as shown in the following discussion (in Section 2). We will also extend the results to multipartite systems $M_{n_{1}} \otimes \cdots \otimes M_{n_{m}}$ in Section 3 .

One may see $[1,3,7,13]$ and their references for some background on linear preserver problems, and the preservers of the Ky Fan $k$-norms and Schatten $p$-norms (without the tensor structure). It was shown in these papers that such norm preservers (except for the Schatten 2-norm preservers) $\phi: M_{n} \rightarrow M_{n}$ have the form

$$
\phi(A)=U A V \quad \text { or } \quad \phi(A)=U A^{t} V
$$

for some unitary matrices $U, V \in M_{n}$, where $A^{t}$ is the transpose of $A$. One can also see $[5,6,10,14]$ and their references for some recent results on linear preserver problems on tensor spaces arising in quantum information science. 
In our discussion, we will use $X^{t}$ and $X^{*}$ to denote the transpose and the conjugate transpose of a square matrix $X$, respectively. For any $A \in M_{m}$ and $B \in M_{n}$, we denote by $A \oplus B$ their direct sum. The $n \times n$ identity matrix will be denoted by $I_{n}$. Denote by $E_{i j}$ the square matrix which the $(i, j)$-entry is equal to one and all the others are equal to zero, where the size of $E_{i j}$ should be clear in the context.

\section{Bipartite systems.}

2.1. Spectral norm. In what follows we denote by $\|\cdot\|$ the spectral norm. Recall that the spectral norm is the same as Ky Fan 1-norm. We first present the result for spectral norm.

THEOREM 2.1. The following are equivalent for a linear map $\phi: M_{m n} \rightarrow M_{m n}$.

(a) $\|\phi(A \otimes B)\|=\|A \otimes B\|$ for all $A \in M_{m}$ and $B \in M_{n}$.

(b) There are unitary matrices $U, V \in M_{m n}$ such that

$$
\phi(A \otimes B)=U\left(\varphi_{1}(A) \otimes \varphi_{2}(B)\right) V \quad \text { for all } A \in M_{m} \text { and } B \in M_{n},
$$

where $\varphi_{s}$ is the identity map or the transposition map $X \mapsto X^{t}$ for $s=1,2$.

Proof. The implication (b) $\Rightarrow$ (a) is obvious. Conversely, assume that $\|\phi(A \otimes B)\|=\|A \otimes B\|$ for all $A \in M_{m}$ and $B \in M_{n}$. In the following, we first show that $\phi$ maps the matrix $E_{i i} \otimes E_{j j}$ to $U\left(E_{i i} \otimes E_{j j}\right) V$ for all $1 \leq i \leq m$ and $1 \leq j \leq n$, where $U$ and $V$ are some unitary matrices.

Let $1 \leq i \leq m$ and $1 \leq j, s \leq n$ with $j \neq s$. Then $\phi\left(E_{i i} \otimes E_{j j}\right)$ has norm one and the same is true for $\phi\left(E_{i i} \otimes E_{j j}+\gamma E_{i i} \otimes E_{s s}\right)$ whenever $|\gamma| \leq 1$. Suppose $x_{j}, y_{j} \in \mathbb{C}^{m n}$ are the left and right norm attaining unit vectors of $\phi\left(E_{i i} \otimes E_{j j}\right)$, i.e., $x_{j}^{*} \phi\left(E_{i i} \otimes E_{j j}\right) y_{j}=1$. Then for any unitary matrices $X_{j}$ and $Y_{j}$ with $x_{j}$ and $y_{j}$ as their first columns, we have $X_{j}^{*} \phi\left(E_{i i} \otimes E_{j j}\right) Y_{j}=[1] \oplus G_{j}$ for some $G_{j} \in M_{m n-1}$ with $\left\|G_{j}\right\| \leq 1$. Since $\phi\left(E_{i i} \otimes E_{j j}+\gamma E_{i i} \otimes E_{s s}\right)$ has norm one for all $|\gamma| \leq 1$, the matrix $X_{j}^{*} \phi\left(E_{i i} \otimes E_{s s}\right) Y_{j}$ must have the form [0] $\oplus G_{s}$ for some $G_{s} \in M_{m n-1}$. It follows that the left and right norm attaining vectors of $\phi\left(E_{i i} \otimes E_{s s}\right)$, say $x_{s}$ and $y_{s}$, must be orthogonal to $x_{j}$ and $y_{j}$ respectively. As $j$ and $s$ are arbitrary, it follows that $\left\{x_{1}, \ldots, x_{n}\right\}$ and $\left\{y_{1}, \ldots, y_{n}\right\}$ are orthonormal sets.

Let $U_{i}$ be an $m n \times m n$ unitary matrix with $x_{1}, \ldots, x_{n}$ as its first $n$ columns and $V_{i}$ be an $m n \times m n$ unitary matrix with $y_{1}^{*}, \ldots, y_{n}^{*}$ as its first $n$ rows. From the above discussion, one has

$$
\phi\left(E_{i i} \otimes E_{j j}\right)=U_{i}\left(E_{j j} \oplus P_{i j}\right) V_{i} \quad \text { for all } j=1, \ldots, n
$$

for some $P_{i j} \in M_{m n-n}$ and hence

$$
\phi\left(E_{i i} \otimes D\right)=U_{i}\left(D \oplus P_{i, D}\right) V_{i} \text { for any diagonal matrix } D \in M_{n}
$$

for some $P_{i, D} \in M_{m n-n}$. Note also that if $1 \leq i, r \leq m$ with $i \neq r$, then $\phi\left(E_{i i} \otimes D+\gamma E_{r r} \otimes D\right)$ has norm one for any diagonal unitary matrix $D \in M_{n}$ and any scalar $\gamma$ with $|\gamma| \leq 1$. Consequently, we see that the left and right norm attaining vectors of $\phi\left(E_{i i} \otimes D\right)$ and those of $\phi\left(E_{r r} \otimes D\right)$ are orthogonal. By a similar argument, there are unitary $U, V \in M_{m n}$ such that

$$
\phi\left(E_{i i} \otimes D\right)=U\left(E_{i i} \otimes D\right) V \quad \text { for any } 1 \leq i \leq m \text { and unitary diagonal } D \in M_{n} .
$$


In particular,

$$
\phi\left(E_{i i} \otimes E_{j j}\right)=U\left(E_{i i} \otimes E_{j j}\right) V \quad \text { for } 1 \leq i \leq m \text { and } 1 \leq j \leq n .
$$

For the sake of the simplicity, we assume that $U$ and $V$ are identity matrices. Next, we show that $\phi\left(E_{i i} \otimes B\right)=E_{i i} \otimes \varphi_{i}(B)$ for all $B \in M_{n}$, where $\varphi_{i}$ is a linear map on $M_{n}$. For any unitary $Y \in M_{n}$ and $1 \leq i \leq m, 1 \leq j \leq n$ we can apply the argument in the preceding paragraphs to conclude that $\phi\left(E_{i i} \otimes Y E_{j j} Y^{*}\right)$ has rank one. Since

$$
\left\|\phi\left(E_{i i} \otimes I_{n}\right)+\gamma \phi\left(E_{i i} \otimes Y E_{j j} Y^{*}\right)\right\|=\left\|\phi\left(E_{i i} \otimes I_{n}+\gamma E_{i i} \otimes Y E_{j j} Y^{*}\right)\right\|=1+\gamma
$$

for any positive scalar $\gamma$, the left and right norm attaining vectors of $\phi\left(E_{i i} \otimes Y E_{j j} Y^{*}\right)$ are also left and right norm attaining vectors of $\phi\left(E_{i i} \otimes I_{n}\right)=E_{i i} \otimes I_{n}$. Thus, $\phi\left(E_{i i} \otimes Y E_{j j} Y^{*}\right)=E_{i i} \otimes Z$ for some rank one $Z \in M_{n}$. Since this is true for any unitary $Y \in M_{n}$, by linearity of $\phi$, we conclude that there exists a linear map $\varphi_{i}: M_{n} \rightarrow M_{n}$ such that

$$
\phi\left(E_{i i} \otimes B\right)=E_{i i} \otimes \varphi_{i}(B) \quad \text { for all } B \in M_{n} .
$$

Clearly, $\varphi_{i}$ preserves the spectral norm. Therefore, $\varphi_{i}$ has the form $X \mapsto W_{i} X \tilde{W}_{i}$ or $X \mapsto W_{i} X^{t} \tilde{W}_{i}$ for some unitary $W_{i}, \tilde{W}_{i} \in M_{n}$ (e.g., see [3] and its references). For simplicity, we may assume that $W_{i}=\tilde{W}_{i}=I_{n}$ for all $i=1, \ldots, m$.

Let $X \in M_{m}$ be any unitary matrix. Repeating the same argument as above, one can show that

$$
\phi\left(X E_{i i} X^{*} \otimes B\right)=U_{X}\left(E_{i i} \otimes \varphi_{i, X}(B)\right) V_{X}
$$

for $1 \leq i \leq m$ and $B \in M_{n}$, where $U_{X}, V_{X} \in M_{m n}$ are unitary matrices depending on $X$ and $\varphi_{i, X}: M_{n} \rightarrow M_{n}$ is either the identity map or the transposition map depending on $i$ and $X$. Moreover, since $\phi\left(I_{m n}\right)=I_{m n}$, we have $V_{X}=U_{X}^{*}$.

Now we show that all the maps $\varphi_{i, X}$ are the same. For any real symmetric $S \in M_{n}$ and any unitary $X \in M_{m}$ we have

$$
\phi\left(I_{m} \otimes S\right)=\phi\left(\sum_{i=1}^{m} X E_{i i} X^{*} \otimes S\right)=U_{X}\left(\sum_{i=1}^{m} X E_{i i} X^{*} \otimes S\right) U_{X}^{*}=U_{X}\left(I_{m} \otimes S\right) U_{X}^{*} .
$$

In particular, when $X=I_{m}$, we have $\phi\left(I_{m} \otimes S\right)=I_{m} \otimes S$. Thus, $U_{X}\left(I_{m} \otimes S\right) U_{X}^{*}=I_{m} \otimes S$ and this yields that $U_{X}$ commutes with $I_{m} \otimes S$ for all real symmetric $S$. Hence, $U_{X}$ has the form $W_{X} \otimes I_{n}$ for some unitary $W_{X} \in M_{m}$ and

$$
\phi\left(X E_{i i} X^{*} \otimes B\right)=\left(W_{X} E_{i i} W_{X}^{*}\right) \otimes \varphi_{i, X}(B) \quad \text { for } \quad 1 \leq i \leq m \text { and } B \in M_{n} .
$$

Now, consider the linear maps $\operatorname{tr}_{1}: M_{m n} \rightarrow M_{n}$ and $\operatorname{Tr}_{1}: M_{m n} \rightarrow M_{n}$ defined by

$$
\operatorname{tr}_{1}(A \otimes B)=(\operatorname{tr} A) B \quad \text { and } \quad \operatorname{Tr}_{1}(A \otimes B)=\operatorname{tr}_{1}(\phi(A \otimes B))
$$

for all $A \in M_{m}$ and $B \in M_{n}$. Notice that the map $\operatorname{tr}_{1}$ is known as the partial trace function in quantum information science context. Then

$$
\operatorname{Tr}_{1}\left(\phi\left(X E_{i i} X^{*} \otimes B\right)\right)=\varphi_{i, X}(B) .
$$


So, $\operatorname{Tr}_{1}$ induces a map $X E_{i i} X^{*} \mapsto \varphi_{i, X}$, where $\varphi_{i, X}$ is either the identity map or the transpose map. Note that $\operatorname{Tr}_{1}$ is linear and therefore continuous, and the set

$$
\left\{X E_{i i} X^{*}: 1 \leq i \leq m, X^{*} X=I_{m}\right\}=\left\{x x^{*} \in M_{m}: x^{*} x=1\right\}
$$

is connected. So, all the maps $\varphi_{i, X}$ have to be the same. Replacing $\phi$ by the map $A \otimes B \mapsto \phi\left(A \otimes B^{t}\right)$, if necessary, we may assume that this common map is the identity map. Next, using the linearity of $\phi$, one can conclude that for every $A \in M_{m}$ and $B \in M_{n}$ we have

$$
\phi(A \otimes B)=\varphi_{1}(A) \otimes B,
$$

where $\varphi_{1}(A) \in M_{m}$ depends on $A$ only. Recall that $\varphi_{1}: M_{m} \rightarrow M_{m}$ is a linear map and $\left\|\varphi_{1}(A)\right\|=$ $\|A\|$ for all $A \in M_{m}$. Hence, $\varphi_{1}$ has the form $A \mapsto U A V$ or $A \mapsto U A^{t} V$ for some unitary $U, V \in M_{m}$. This completes the proof.

2.2. Ky Fan $k$-norms. We now turn to Ky Fan $k$-norms. Two matrices $A, B \in M_{n}$ are called orthogonal if $A B^{*}=A^{*} B=0$ (see [16]). We write $A \perp B$ to indicate that $A$ and $B$ are orthogonal. It is shown in [16] that $A \perp B$ if and only if there are unitary matrices $U, V \in M_{n}$ such that $U A V=\operatorname{diag}\left(a_{1}, \ldots, a_{n}\right)$ and $U B V=\operatorname{diag}\left(b_{1}, \ldots, b_{n}\right)$ with $a_{i}, b_{i} \geq 0$ and $a_{i} b_{i}=0$ for $i=1, \ldots, n$. The matrices $A_{1}, \ldots, A_{t}$ are said to be pairwise orthogonal if $A_{i}^{*} A_{j}=A_{i} A_{j}^{*}=0$ for any distinct $i, j \in\{1, \ldots, t\}$. In this case, there are unitary matrices $U, V \in M_{n}$ such that $U A_{i} V=D_{i}$ for $i=1, \ldots, t$ with each $D_{i}$ being nonnegative diagonal matrix and $D_{i} D_{j}=0$ for any distinct $i, j \in\{1, \ldots, t\}$.

We have the following lemmas relating to orthogonality, which are useful in the proof of Ky Fan $k$-norm results (Theorems 2.6 and 3.1).

Lemma 2.2. [16] Let $A, B \in M_{n}$ be nonzero matrices. Then

$$
\|\alpha A+\beta B\|_{(k)}=|\alpha|\|A\|_{(k)}+|\beta|\|B\|_{(k)}
$$

for every pair of complex numbers $\alpha$ and $\beta$ if and only if $A \perp B$ and $\operatorname{rank} A+\operatorname{rank} B \leq k$.

Denote by $\sigma(A)$ the spectrum of a matrix $A \in M_{n}$. Using the same arguments as in the proof of Lemma 2 in [16], we have the following result. (One can also see [8, p.468, Problem 3] for part (a) of Lemma 2.3.)

Lemma 2.3. Let $A \in M_{n}$ be positive semidefinite and let $B \in M_{n}$ be Hermitian.

(a) $\sigma(A B) \subseteq \mathbb{R}$.

(b) If $\sigma(A B)=\{0\}$, then there exists a unitary $U \in M_{n}$ such that

$$
U A U^{*}=\left[\begin{array}{cc}
A_{1} & 0 \\
0 & 0
\end{array}\right] \quad \text { and } \quad U B U^{*}=\left[\begin{array}{cc}
0 & X \\
X^{*} & B_{1}
\end{array}\right]
$$

where $A_{1} \in M_{s}$ is invertible and $B_{1} \in M_{n-s}$ with $0 \leq s \leq n$.

Lemma 2.4. Let $1 \leq k \leq n$ and $A, B \in M_{n}$ with spectral norm at most 1 . Suppose

$$
\operatorname{rank} A \leq k, \quad\|A+\alpha B\|_{(k)}=k \quad \text { and } \quad \underset{5}{\|2 A+\alpha B\|_{(k)}}=\|A\|_{(k)}+\|A+\alpha B\|_{(k)}
$$


for any unit complex number $\alpha$. Then $A \perp B$ and $\sigma\left(A^{*} A\right) \subseteq\{0,1\}$.

Proof. First, let $\alpha=1$ and suppose $2 A+B$ has singular value decomposition $2 A+B=U_{1} D V_{1}$ with $D=\operatorname{diag}\left(d_{1}, \ldots, d_{n}\right)$ and $d_{1} \geq \cdots \geq d_{n} \geq 0$. Then

$$
D=U_{1}^{*}(2 A+B) V_{1}^{*}=U_{1}^{*} A V_{1}^{*}+U_{1}^{*}(A+B) V_{1}^{*} .
$$

Denote the diagonal entries of $U_{1}^{*} A V_{1}^{*}$ and $U_{1}^{*} B V_{1}^{*}$ by $a_{1}, \ldots, a_{n}$ and $b_{1}, \ldots, b_{n}$, respectively. Then we have

$$
\sum_{i=1}^{k}\left|a_{i}\right| \leq\|A\|_{(k)}, \quad \sum_{i=1}^{k}\left|a_{i}+b_{i}\right| \leq\|A+B\|_{(k)}, \quad \sum_{i=1}^{k}\left(2 a_{i}+b_{i}\right)=\sum_{i=1}^{k} d_{i}=\|2 A+B\|_{(k)} .
$$

It follows that

$$
\sum_{i=1}^{k}\left(a_{i}+\left(a_{i}+b_{i}\right)\right) \leq \sum_{i=1}^{k}\left(\left|a_{i}\right|+\left|a_{i}+b_{i}\right|\right) \leq\|A\|_{(k)}+\|A+B\|_{(k)}=\|2 A+B\|_{(k)}=\sum_{i=1}^{k}\left(2 a_{i}+b_{i}\right),
$$

and so all the above inequalities are indeed equalities, which ensure that $a_{i}=\left|a_{i}\right|$ and $a_{i}+b_{i}=\left|a_{i}+b_{i}\right|$ are nonnegative real numbers with

$$
\sum_{i=1}^{k} a_{i}=\sum_{i=1}^{k}\left|a_{i}\right|=\|A\|_{(k)} \quad \text { and } \quad \sum_{i=1}^{k}\left(a_{i}+b_{i}\right)=\sum_{i=1}^{k}\left|a_{i}+b_{i}\right|=\|A+B\|_{(k)} .
$$

By Theorem 3.1 of [12], we have

$$
U_{1}^{*} A V_{1}^{*}=A_{1} \oplus A_{2} \quad \text { and } \quad U_{1}^{*}(A+B) V_{1}^{*}=\left(A_{1}+B_{1}\right) \oplus\left(A_{2}+B_{2}\right),
$$

where $A_{1}$ and $A_{1}+B_{1}$ are $k \times k$ positive semidefinite matrices and

$$
\|A\|_{(k)}=\left\|A_{1}\right\|_{(k)}=\operatorname{tr} A_{1}, \quad\|A+B\|_{(k)}=\left\|A_{1}+B_{1}\right\|_{(k)}=\operatorname{tr}\left(A_{1}+B_{1}\right) .
$$

Since $\operatorname{rank} A \leq k$, it follows that $A_{2}=0$ and we may assume that $B_{2}=\operatorname{diag}\left(d_{k+1}, \ldots, d_{n}\right)$. Without loss of generality, we also assume that $U_{1}=V_{1}=I_{n}$ and

$$
A=A_{1} \oplus 0, \quad B=B_{1} \oplus \operatorname{diag}\left(d_{k+1}, \ldots, d_{n}\right) .
$$

Now take a unit $\alpha_{1} \neq \pm 1$. Suppose the singular value decomposition of $2 A_{1}+\alpha_{1} B_{1}$ is $2 A_{1}+$ $\alpha_{1} B_{1}=U_{2} D_{1} V_{2}$ with $D_{1}=\operatorname{diag}\left(\beta_{1}, \ldots, \beta_{k}\right)$ and $\beta_{1} \geq \cdots \geq \beta_{k} \geq 0$. Let $U_{3}=U_{2}^{*} \oplus I_{n-k}$ and $V_{3}=V_{2}^{*} \oplus \alpha_{1}^{-1} I_{n-k}$. Set $A_{3}=U_{2}^{*} A_{1} V_{2}^{*}$ and $B_{3}=\alpha_{1} U_{2}^{*} B_{1} V_{2}^{*}$. Then

$$
\begin{aligned}
\operatorname{diag}\left(\beta_{1}, \ldots, \beta_{k}, d_{k+1}, \ldots, d_{n}\right) & =U_{3}\left(2 A+\alpha_{1} B\right) V_{3} \\
& =U_{2}^{*} A_{1} V_{2}^{*} \oplus 0_{n-k}+U_{2}^{*}\left(A_{1}+\alpha_{1} B_{1}\right) V_{2}^{*} \oplus \operatorname{diag}\left(d_{k+1}, \ldots, d_{n}\right) \\
& =A_{3} \oplus 0_{n-k}+\left(A_{3}+B_{3}\right) \oplus \operatorname{diag}\left(d_{k+1}, \ldots, d_{n}\right) .
\end{aligned}
$$

First, assume that $\left\|2 A+\alpha_{1} B\right\|_{(k)}=\sum_{i=1}^{k} \beta_{i}$. Using the same argument as above, we see that $A_{3}$ and $A_{3}+B_{3}$ are $k \times k$ positive semidefinite matrices with $\|A\|_{(k)}=\operatorname{tr} A_{3}$ and $\left\|A+\alpha_{1} B\right\|_{(k)}=\operatorname{tr}\left(A_{3}+B_{3}\right)$. By Lemma $2.3(\mathrm{a}), \sigma\left(A_{1} B_{1}\right) \subseteq \mathbb{R}$ and $\sigma\left(A_{3} B_{3}\right) \subseteq \mathbb{R}$. Also

$$
\sigma\left(\alpha_{1} A_{3} B_{3}\right)=\sigma\left(\alpha_{1} A_{3} B_{3}^{*}\right)=\sigma\left(\alpha_{1}\left(U_{2}^{*} A_{1} V_{2}^{*}\right)\left(\alpha_{1} U_{2}^{*} B_{1} V_{2}\right)^{*}\right)=\sigma\left(U_{2}^{*} A_{1} B_{1} U_{2}\right)=\sigma\left(A_{1} B_{1}\right) \subseteq \mathbb{R} .
$$


This implies $\sigma\left(A_{1} B_{1}\right)=\sigma\left(A_{3} B_{3}\right)=\{0\}$. According to Lemma 2.3(b), we know that there exists a unitary $U_{4}$ such that

$$
U_{4} A_{3} U_{4}^{*}=A_{4} \oplus 0, \quad U_{4} B_{3} U_{4}^{*}=\left[\begin{array}{cc}
0 & X \\
X^{*} & B_{4}
\end{array}\right],
$$

where $A_{4} \in M_{s}$ is positive definite for some $0 \leq s \leq k, B_{4} \in M_{k-s}$, and $\left\|A_{3}+B_{3}\right\|_{(k)}=\operatorname{tr} A_{4}+\operatorname{tr} B_{4}$. Since both $A$ and $B$ have spectral norm at most 1, the diagonal entries of $A_{4}$ and $B_{4}$ are less than or equal to one. So $\operatorname{tr}\left(A_{3}+B_{3}\right)=\left\|A_{3}+B_{3}\right\|_{(k)}=\left\|A+\alpha_{1} B\right\|_{(k)}=k$ ensures that all the diagonal entries of $A_{4}$ and $B_{4}$ are equal to one and that the singular values of $A_{3}+B_{3}$ are all equal to one. It follows that $X=0, A_{4}=I_{s}$, and $B_{4}=I_{k-s}$, which implies $A \perp B$ and $\sigma\left(A^{*} A\right) \subseteq\{0,1\}$.

Now, assume that $\left\|2 A+\alpha_{1} B\right\|_{(k)} \neq \sum_{i=1}^{k} \beta_{i}$ and suppose that the largest $k$ singular values of $2 A+\alpha_{1} B$ are $\beta_{1}, \ldots, \beta_{r}, d_{k+1}, \ldots, d_{k+s}$ with $r+s=k$ and $r<k$. Denote by $\tilde{A}=A_{3} \oplus 0$ and $\tilde{B}=B_{3} \oplus \operatorname{diag}\left(d_{k+1}, \ldots, d_{n}\right)$. Applying Theorem 3.1 of [12] again, it follows that

$$
\tilde{A}=\tilde{A}[1, \ldots, r] \oplus 0_{n-r} \geq 0 \quad \text { and } \quad \tilde{B}=\tilde{B}[1, \ldots, r] \oplus \operatorname{diag}\left(\beta_{r+1}, \ldots, \beta_{k}, d_{k+1}, \ldots, d_{n}\right) \geq 0,
$$

where $\tilde{A}[1, \ldots, r]$ and $\tilde{B}[1, \ldots, r]$ are the principal submatrices of $\tilde{A}$ and $\tilde{B}$ indexed by $1, \ldots, r$. Without loss of generality, we can assume

$$
A=\tilde{A}[1, \ldots, r] \oplus 0_{n-r} \quad \text { and } \quad B=\tilde{B}[1, \ldots, r] \oplus \operatorname{diag}\left(\beta_{r+1}, \ldots, \beta_{k}, d_{k+1}, \ldots, d_{n}\right) .
$$

Choosing a unit $\alpha_{2} \neq\left\{ \pm 1, \pm \alpha_{1}\right\}$ and repeating the above process at most $n-2$ times, we can show that $A \perp B$ and $\sigma\left(A^{*} A\right) \subseteq\{0,1\}$.

Lemma 2.5. Let $1 \leq k \leq n$ and $A, B \in M_{n}$ such that $A$ has rank at least $k$ and $\sigma\left(A^{*} A\right) \subseteq\{0,1\}$. If

$$
\|A+\alpha B\|_{(k)}=k
$$

for all unit complex numbers $\alpha$, then $A \perp B$.

Proof. Without loss of generality, we may assume that $A=I_{s} \oplus 0$ with $s \geq k$. Denote $A=\left[a_{i j}\right]$ and $B=\left[b_{i j}\right]$. We claim that for any arbitrary and fixed $1 \leq p_{1}<\cdots<p_{k} \leq s, b_{i j}=0$ whenever $\{i, j\} \cap\left\{p_{1}, \ldots, p_{k}\right\} \neq \emptyset$. If the claim holds, then $B$ has the from $0_{s} \oplus B_{2}$ and therefore $A \perp B$. To prove the claim, it suffices to show the case when $\left(p_{1}, \ldots, p_{k}\right)=(1, \ldots, k)$. Notice that

$$
k=\|A+\alpha B\|_{(k)} \geq \sum_{j=1}^{k}\left|a_{j j}+\alpha b_{j j}\right| \geq\left|\sum_{j=1}^{k}\left(a_{j j}+\alpha b_{j j}\right)\right|=\left|k+\alpha \sum_{j=1}^{k} b_{j j}\right| .
$$

The two equalities holds for all complex unit $\alpha$ if and only if $b_{j j}=0$ for all $1 \leq j \leq k$. Thus, we have $\|A+\alpha B\|_{(k)}=k=\sum_{j=1}^{k}\left|a_{j j}+\alpha b_{j j}\right|$ for all complex unit $\alpha$. Now by [12, Theorem 3.1],

$$
A+\alpha B=C_{\alpha} \oplus D_{\alpha}
$$

where $C_{\alpha}$ is positive semidefinite and $\left\|C_{\alpha}\right\|_{(k)}=k$. It follows that $B=B_{1} \oplus B_{2}$ with $B_{1} \in M_{k}$. Notice that $C_{\alpha}=I_{k}+\alpha B_{1}$ is positive semidefinite for all complex unit $\alpha$. Then $B_{1}$ has to be the zero matrix, i.e., $B=0_{k} \oplus B_{2}$. Therefore, the claim holds and the result follows. 
We now present of the result for the Ky Fan $k$-norm.

TheOREM 2.6. Let $m, n \geq 2$ and $2 \leq k \leq m n$ and $\phi: M_{m n} \rightarrow M_{m n}$ be a linear map. The following are equivalent.

(a) $\|\phi(A \otimes B)\|_{(k)}=\|A \otimes B\|_{(k)}$ for all $A \in M_{m}$ and $B \in M_{n}$.

(b) There are unitary matrices $U, V \in M_{m n}$ such that

$$
\phi(A \otimes B)=U\left(\varphi_{1}(A) \otimes \varphi_{2}(B)\right) V \quad \text { for all } \quad A \in M_{m} \text { and } B \in M_{n},
$$

where $\varphi_{s}$ is the identity map or the transposition map $X \mapsto X^{t}$ for $s=1,2$.

Proof. The implication (b) $\Rightarrow$ (a) is obvious. Conversely, assume that $\|\phi(A \otimes B)\|_{(k)}=\|A \otimes B\|_{(k)}$ for all $A \in M_{m}$ and $B \in M_{n}$. We assert that there exist unitary $U, V \in M_{m n}$ such that

$$
\phi\left(E_{i i} \otimes E_{j j}\right)=U\left(E_{i i} \otimes E_{j j}\right) V \quad \text { for } 1 \leq i \leq m \text { and } 1 \leq j \leq n .
$$

It suffices to show that

$$
\phi\left(E_{i i} \otimes E_{j j}\right) \perp \phi\left(E_{r r} \otimes E_{s s}\right)
$$

for any distinct pairs $(i, j)$ and $(r, s)$ with $1 \leq i, r \leq m$ and $1 \leq j, s \leq n$. We distinguish three cases.

Case 1. Suppose $i=r$ or $j=s$. We have

$$
\left\|\alpha \phi\left(E_{i i} \otimes E_{j j}\right)+\beta \phi\left(E_{r r} \otimes E_{s s}\right)\right\|_{(k)}=|\alpha|\left\|\phi\left(E_{i i} \otimes E_{j j}\right)\right\|_{(k)}+|\beta|\left\|\phi\left(E_{r r} \otimes E_{s s}\right)\right\|_{(k)}
$$

for all complex numbers $\alpha$ and $\beta$. Applying Lemma 2.2, we have (2.2) and

$$
\operatorname{rank} \phi\left(E_{i i} \otimes E_{j j}\right)+\operatorname{rank} \phi\left(E_{r r} \otimes E_{s s}\right) \leq k .
$$

Case 2. Suppose $i \neq r$ and $j \neq s$. Let

$$
G=\phi\left(E_{i i} \otimes\left(E_{j j}+E_{s s}\right)\right) \quad \text { and } \quad H=\phi\left(E_{r r} \otimes\left(E_{j j}+E_{s s}\right)\right) .
$$

Subcase 2.a. Assume first that $k \leq 3$. By Case $1, \phi\left(E_{i i} \otimes E_{j j}\right)$ and $\phi\left(E_{i i} \otimes E_{s s}\right)$ are orthogonal with $\left\|\phi\left(E_{i i} \otimes E_{j j}\right)\right\|_{(k)}=\left\|\phi\left(E_{i i} \otimes E_{s s}\right)\right\|_{(k)}=1$. So, $\|G\| \leq 1$ and $\operatorname{rank} G \leq k$. Similarly, $\|H\| \leq 1$ and $\operatorname{rank} H \leq k$. Furthermore, $\|G+\gamma H\|_{(k)}=k$, and $\|2 G+\gamma H\|_{(k)}=\|G\|_{(k)}+\|G+\gamma H\|_{(k)}$ for all complex units $\gamma$. Applying Lemma 2.4, we get $G \perp H$. Because $\phi\left(E_{i i} \otimes E_{j j}\right) \perp \phi\left(E_{i i} \otimes E_{s s}\right)$ and $\phi\left(E_{r r} \otimes E_{j j}\right) \perp \phi\left(E_{r r} \otimes E_{s s}\right)$, we have (2.2).

Subcase 2.b. Now, suppose that $k>3$. We have

$$
\|\alpha G+\beta H\|_{(k)}=|\alpha|\|G\|_{(k)}+|\beta|\|H\|_{(k)}
$$

for all complex numbers $\alpha, \beta$. Applying Lemma 2.2 again, we get $G \perp H$ and, hence, (2.2) follows.

From above, we showed that (2.1) holds. For the sake of the simplicity, we assume that $U$ and $V$ are identity matrices. Now, for any unitary $Y \in M_{n}$ and $1 \leq i \leq m, 1 \leq j \leq n$ we can apply the argument in the preceding paragraphs to conclude that $\phi\left(E_{i i} \otimes Y E_{j j} Y^{*}\right)$ has rank one and it is orthogonal to $\phi\left(E_{r r} \otimes Y E_{s s} Y^{*}\right)$ for any distinct pairs $(i, j)$ and $(r, s)$. It follows that

$$
\left\|\phi\left(E_{i i} \otimes I_{n}\right)+\gamma \phi\left(E_{i i} \otimes Y E_{j j} Y^{*}\right)\right\|=\underset{8}{\left\|\phi\left(E_{i i} \otimes I_{n}+\gamma E_{i i} \otimes Y E_{j j} Y^{*}\right)\right\|=1+\gamma}
$$


for any positive scalar $\gamma$. Thus, $\phi\left(E_{i i} \otimes Y E_{j j} Y^{*}\right)=E_{i i} \otimes Z$ for some rank one $Z \in M_{n}$. Since this is true for any $1 \leq i \leq m$ and unitary $Y \in M_{n}$, we conclude that there exists a linear map $\varphi_{i}: M_{n} \rightarrow M_{n}$ such that

$$
\phi\left(E_{i i} \otimes B\right)=E_{i i} \otimes \varphi_{i}(B)
$$

for all complex matrices $B \in M_{n}$. Clearly, $\varphi_{i}$ preserves the Ky Fan $k$-norm of all $B \in M_{n}$ and $\varphi_{i}\left(E_{j j}\right)=E_{j j}$ for all $1 \leq j \leq n$. (Here the Ky Fan $k$-norm reduces to the trace norm if $k \geq n$.) Hence, $\varphi_{i}$ has the form $X \mapsto W_{i} X \tilde{W}_{i}$ or $X \mapsto W_{i} X^{t} \tilde{W}_{i}$ for some unitary $W_{i}, \tilde{W}_{i} \in M_{n}$. Now, we can adapt the arguments in the last two paragraphs in the proof of Theorem 2.1 to obtain our conclusion.

2.3. Schatten $p$-norms. We now study the linear preserver for Schatten $p$-norms. We first present a key lemma of the result.

Lemma 2.7. [17] Let $T, S \in M_{n}$. Then

(i) $2^{p-1}\left(\|T\|_{p}^{p}+\|S\|_{p}^{p}\right) \leq\|T+S\|_{p}^{p}+\|T-S\|_{p}^{p} \leq 2\left(\|T\|_{p}^{p}+\|S\|_{p}^{p}\right)$ if $1 \leq p \leq 2$,

(ii) $2\left(\|T\|_{p}^{p}+\|S\|_{p}^{p}\right) \leq\|T+S\|_{p}^{p}+\|T-S\|_{p}^{p} \leq 2^{p-1}\left(\|T\|_{p}^{p}+\|S\|_{p}^{p}\right)$ if $2 \leq p<\infty$. If $p=2$, all equalities always hold; if $p \neq 2$, any equality holds if and only if $T^{*} T S^{*} S=S^{*} S T^{*} T=0$.

REMARK 2.8. Suppose $p \neq 2$ and the singular values of $T$ and $S$ are $\left\{t_{1}, \ldots, t_{n}\right\}$ and $\left\{s_{1}, \ldots, s_{n}\right\}$, respectively. If any of equality in Lemma 2.7 holds, then $T^{*} T S^{*} S=S^{*} S T^{*} T=0$ implies $T^{*} T \perp S^{*} S$. So there exists unitary $U \in M_{n}$ such that $U^{*} T^{*} T U=\operatorname{diag}\left(t_{1}^{2}, \ldots, t_{n}^{2}\right)$ and $U^{*} S^{*} S U=\operatorname{diag}\left(s_{1}^{2}, \ldots, s_{n}^{2}\right)$ with $s_{i} t_{i}=0$ for $i=1, \ldots, n$. Replacing $T$ and $S$ with $T^{*}$ and $S^{*}$, we get $T T^{*} S S^{*}=0$ and there exists unitary $V \in M_{n}$ such that $V^{*} T T^{*} V=\operatorname{diag}\left(t_{1}^{2}, \ldots, t_{n}^{2}\right)$ and $V^{*} S S^{*} V=\operatorname{diag}\left(s_{1}^{2}, \ldots, s_{n}^{2}\right)$. It follows that $V^{*} T U=\operatorname{diag}\left(t_{1}, \ldots, t_{n}\right)$ and $V^{*} S U=\operatorname{diag}\left(s_{1}, \ldots, s_{n}\right)$, which implies $T \perp S$.

TheOrem 2.9. Let $1 \leq p<\infty$ and $p \neq 2$ and $\phi: M_{m n} \rightarrow M_{m n}$ be a linear map. Then the following are equivalent.

(a) $\|\phi(A \otimes B)\|_{p}=\|A \otimes B\|_{p}$ for all $A \in M_{m}$ and $B \in M_{n}$.

(b) There are unitary matrices $U, V \in M_{m n}$ such that

$$
\phi(A \otimes B)=U\left(\varphi_{1}(A) \otimes \varphi_{2}(B)\right) V \quad \text { for all } A \in M_{m} \text { and } B \in M_{n},
$$

where $\varphi_{s}$ is the identity map or the transposition map $X \mapsto X^{t}$ for $s=1,2$.

Proof. The implication (b) $\Rightarrow$ (a) is obvious. Conversely, assume that $\|\phi(A \otimes B)\|_{p}=\|A \otimes B\|_{p}$ for all $A \otimes B \in M_{m n}$. We first conclude there exist unitary $U, V \in M_{m n}$ such that

$$
\phi\left(E_{i i} \otimes E_{j j}\right)=U\left(E_{i i} \otimes E_{j j}\right) V \quad \text { for } 1 \leq i \leq m \text { and } 1 \leq j \leq n .
$$

Then we can adapt the arguments in the last three paragraphs in the proof of Theorem 2.1 to verify that $\phi$ has the form claimed in (b).

As in the proof of Theorem 2.6, to prove (2.4), it suffices to show that

$$
\phi\left(E_{i i} \otimes E_{j j}\right) \perp \phi\left(E_{r r} \otimes E_{s s}\right)
$$

for any distinct pairs $(i, j)$ and $(r, s)$ with $1 \leq i, r \leq m$ and $1 \leq j, s \leq n$. 
If $i=r$ or $j=s$, we have

$\left\|\phi\left(E_{i i} \otimes E_{j j}\right)+\phi\left(E_{r r} \otimes E_{s s}\right)\right\|_{p}^{p}+\left\|\phi\left(E_{i i} \otimes E_{j j}\right)-\phi\left(E_{r r} \otimes E_{s s}\right)\right\|_{p}^{p}=2\left(\left\|\phi\left(E_{i i} \otimes E_{j j}\right)\right\|_{p}^{p}+\left\|\phi\left(E_{r r} \otimes E_{s s}\right)\right\|_{p}^{p}\right)$.

Applying Lemma 2.7 and Remark 2.8, we get (2.5). If $i \neq r$ and $j \neq s$, we have

$$
\begin{array}{r}
\left\|\phi\left(E_{i i} \otimes\left(E_{j j}+E_{s s}\right)\right)+\phi\left(E_{r r} \otimes\left(E_{j j}+E_{s s}\right)\right)\right\|_{p}^{p}+\left\|\phi\left(E_{i i} \otimes\left(E_{j j}+E_{s s}\right)\right)-\phi\left(E_{r r} \otimes\left(E_{j j}+E_{s s}\right)\right)\right\|_{p}^{p} \\
=2\left(\left\|\phi\left(E_{i i} \otimes\left(E_{j j}+E_{s s}\right)\right)\right\|_{p}^{p}+\left\|\phi\left(E_{r r} \otimes\left(E_{j j}+E_{s s}\right)\right)\right\|_{p}^{p}\right) .
\end{array}
$$

By Lemma 2.7, we have $\phi\left(E_{i i} \otimes\left(E_{j j}+E_{s s}\right)\right) \perp \phi\left(E_{r r} \otimes\left(E_{j j}+E_{s s}\right)\right)$. With the fact that $\phi\left(E_{i i} \otimes\right.$ $\left.E_{j j}\right) \perp \phi\left(E_{i i} \otimes E_{s s}\right)$ and $\phi\left(E_{r r} \otimes E_{j j}\right) \perp \phi\left(E_{r r} \otimes E_{s s}\right)$, we conclude that (2.5) holds. This completes the proof. $\square$

Remark 2.10. For $p=2$, i.e., the Frobenius norm case, the statements (a) and (b) in Theorem 2.9 are not equivalent. One can consider the linear map $\psi(A)=\left[b_{i j}\right]$ for $A=\left[a_{i j}\right] \in M_{m n}$ such that $b_{1, m n}=a_{m n, 1}, b_{m n, 1}=a_{1, m n}$ and $b_{i j}=a_{i j}$ for $(i, j) \notin\{(1, m n),(m n, 1)\}$. In fact, any linear map on $M_{m n}$ preserving the inner product $(A, B)=\operatorname{tr}\left(A B^{*}\right)$ on $M_{m n}$ will preserve the Frobenius norm.

Remark 2.11. Note that the maps in Theorems 2.1,2.6, and 2.9 may not satisfy $\|\phi(C)\|=\|C\|$ for all $C \in M_{m n}$. For instance, if $\phi(A \otimes B)=A \otimes B^{t}$ and if

$$
C_{r}=r^{2} E_{11} \otimes E_{11}+r\left(E_{12} \otimes E_{12}+E_{21} \otimes E_{21}\right)+E_{22} \otimes E_{22} \quad \text { for } \quad r \geq 0
$$

then $C_{r}$ has singular values $r^{2}+1,0, \ldots, 0$, and $\phi\left(C_{r}\right)$ has singular values $r^{2}, r, r, 1,0, \ldots, 0$. Then for any positive number $r \neq 1,\left\|\phi\left(C_{r}\right)\right\| \neq\left\|C_{r}\right\|$ unless $\|\cdot\|$ is the Frobenious norm. However, if we assume that $\phi$ satisfies $\|\phi(C)\|=\|C\|$ for $C$ of the tensor form $A \otimes B$, and also for $C=C_{r}$ for some positive number $r \neq 1$, then one easily deduces that both $\varphi_{i}$ mentioned in the theorems have to be of the same type, i.e., both are identity map, or both are the transposition map. It follows that there are unitary $U, V \in M_{m n}$ such that $\phi$ has the form

$$
X \mapsto U X V \quad \text { or } \quad X \mapsto U X^{t} V
$$

and hence, $\|\phi(X)\|=\|X\|$ for all $X \in M_{m n}$.

3. Multipartite systems. In this section we extend the previous results to multipartite systems $M_{n_{1}} \otimes \cdots \otimes M_{n_{m}}, m \geq 2$. Let $A_{i} \in M_{n_{i}}, i=1, \ldots, m$. We denote $\bigotimes_{i=1}^{m} A_{i}=A_{1} \otimes A_{2} \otimes \cdots \otimes A_{m}$.

TheOrem 3.1. Let $1 \leq k \leq \prod_{i=1}^{m} n_{i}$ and $\phi: M_{n_{1} \cdots n_{m}} \rightarrow M_{n_{1} \cdots n_{m}}$ be a linear map. The following are equivalent.

(a) $\left\|\phi\left(A_{1} \otimes \cdots \otimes A_{m}\right)\right\|_{(k)}=\left\|A_{1} \otimes \cdots \otimes A_{m}\right\|_{(k)}$ for all $A_{i} \in M_{n_{i}}, i=1, \ldots, m$.

(b) There are unitary matrices $U, V \in M_{n_{1} \cdots n_{m}}$ such that

$$
\phi\left(A_{1} \otimes \cdots \otimes A_{m}\right)=U\left(\varphi_{1}\left(A_{1}\right) \otimes \cdots \otimes \varphi_{m}\left(A_{m}\right)\right) V \quad \text { for all } \quad A_{i} \in M_{n_{i}}, i=1, \ldots, m
$$

where $\varphi_{s}$ is the identity map or the transposition map $X \mapsto X^{t}$ for $s=1, \ldots, m$.

Proof. The sufficiency part is clear. To prove the necessity part, we use induction on $m$. By Theorem 2.1 and Theorem 2.6, we already know that the statement of Theorem 3.1 is true for bipartite systems. So, assume that $m \geq 3$ and that the result holds for all $(m-1)$-partite systems. We need to prove that the same is true for $m$-partite systems. 
Denote $N=\prod_{i=1}^{m} n_{i}$. First we claim that there exist unitary $U, V \in M_{N}$ such that

$$
\phi\left(E_{j_{1} j_{1}} \otimes \cdots \otimes E_{j_{m} j_{m}}\right)=U\left(E_{j_{1} j_{1}} \otimes \cdots \otimes E_{j_{m} j_{m}}\right) V \quad \text { for all } \quad 1 \leq j_{i} \leq n_{i} .
$$

When $k=1$, as in the proof of Theorem 2.1 , we can successively consider

$$
\begin{aligned}
& \phi\left(E_{i_{1} i_{1}} \otimes \cdots \otimes E_{i_{m-2}, i_{m-2}} \otimes E_{i_{m-1}, i_{m-1}} \otimes\right.\left.\left(E_{i_{m} i_{m}}+\gamma E_{j_{m} j_{m}}\right)\right), \\
& \phi\left(E_{i_{1} i_{1}} \otimes \cdots \otimes E_{i_{m-2}, i_{m-2}} \otimes\right.\left.\left(E_{i_{m-1} i_{m-1}}+\gamma E_{j_{m-1} j_{m-1}}\right) \otimes D_{m}\right), \quad \ldots, \\
& \phi\left(\left(E_{i_{1} i_{1}}+\gamma E_{j_{1} j_{1}}\right) \otimes D_{2} \otimes \cdots \otimes D_{m-2} \otimes D_{m-1} \otimes D_{m}\right)
\end{aligned}
$$

to obtain (3.2), where $D_{2}, \ldots, D_{m}$ are arbitrary diagonal matrices.

When $k \geq 2$, as in the proof of Theorem 2.6, it suffices to show

$$
\phi\left(E_{i_{1} i_{1}} \otimes \cdots \otimes E_{i_{m} i_{m}}\right) \perp \phi\left(E_{j_{1} j_{1}} \otimes \cdots \otimes E_{j_{m} j_{m}}\right) \quad \text { for any } \quad\left(i_{1}, \ldots, i_{m}\right) \neq\left(j_{1}, \ldots, j_{m}\right) .
$$

To confirm (3.3), it suffices to verify that for any $1 \leq r \leq m$,

$\phi\left(\bigotimes_{u=1}^{r-1}\left(E_{i_{u} i_{u}}+E_{j_{u} j_{u}}\right) \otimes E_{i_{r} i_{r}} \otimes \bigotimes_{u=r+1}^{m} E_{i_{u} i_{u}}\right) \perp \phi\left(\bigotimes_{u=1}^{r-1}\left(E_{i_{u} i_{u}}+E_{j_{u} j_{u}}\right) \otimes E_{j_{r} j_{r}} \otimes \bigotimes_{u=r+1}^{m} E_{i_{u} i_{u}}\right)$

for any distinct $\mathbf{i}=\left(i_{1}, \ldots, i_{m}\right)$ and $\mathbf{j}=\left(j_{1}, \ldots, j_{m}\right)$ with $i_{u} \neq j_{u}, 1 \leq u \leq r$. Denote by $G_{r}=G_{r}(\mathbf{i}, \mathbf{j})$ and $\hat{G}_{r}=\hat{G}_{r}(\mathbf{i}, \mathbf{j})$ the two matrices in (3.4) accordingly. We consider two cases.

Case 1. For $r \leq \log _{2} k$, as $\left\|\alpha G_{r}+\beta \hat{G}_{r}\right\|_{(k)}=|\alpha|\left\|G_{r}\right\|_{(k)}+|\beta|\left\|\hat{G}_{r}\right\|_{(k)}$ for all complex $\alpha$ and $\beta$. Applying Lemma 2.2, we get

$$
G_{r} \perp \hat{G}_{r} \quad \text { and } \operatorname{rank} G_{r}+\operatorname{rank} \hat{G}_{r} \leq k \text { for all } r \leq \log _{2} k .
$$

Now as $G_{s+1}=G_{s}+\hat{G}_{s}$ and $G_{s} \perp \hat{G}_{s}$ for all $s \leq \log _{2} k$,

$$
\left\|G_{s+1}\right\| \leq \max \left\{\left\|G_{s}\right\|,\left\|\hat{G}_{s}\right\|\right\} \leq \max \left\{\left\|G_{s}(\mathbf{i}, \mathbf{j})\right\|: i_{u} \neq j_{u}, 1 \leq u \leq s\right\},
$$

where $\|\cdot\|$ is the spectral norm. Hence,

$$
\max \left\{\left\|G_{s+1}(\mathbf{i}, \mathbf{j})\right\|: i_{u} \neq j_{u}, 1 \leq u \leq s+1\right\} \leq \max \left\{\left\|G_{s}(\mathbf{i}, \mathbf{j})\right\|: i_{u} \neq j_{u}, 1 \leq u \leq s\right\} .
$$

As the inequality holds for all $s \leq \log _{2} k$, it follows that

$$
\begin{aligned}
\left\|G_{r}\right\| \leq \max \left\{\left\|G_{1}(\mathbf{i}, \mathbf{j})\right\|: i_{1} \neq j_{1}\right\} & =\max \left\{\left\|\phi\left(E_{i_{1} i_{1}} \otimes \cdots \otimes E_{i_{m} i_{m}}\right)\right\|:\left(i_{1}, \ldots, i_{m}\right)\right\} \\
& \leq \max \left\{\left\|\phi\left(E_{i_{1} i_{1}} \otimes \cdots \otimes E_{i_{m} i_{m}}\right)\right\|_{(k)}:\left(i_{1}, \ldots, i_{m}\right)\right\}=1 .
\end{aligned}
$$

Similarly, one conclude that $\left\|\hat{G}_{r}\right\| \leq 1$.

Case 2. For $r>\log _{2} k$, we claim that $G_{r}$ and $\hat{G}_{r}$ are orthogonal and both of them have singular values 0 and 1 only. We prove the claim by induction on $r$. 
Suppose $\log _{2} k<r \leq 1+\log _{2} k$. Notice that $G_{r}=G_{r-1}+\hat{G}_{r-1}$. By Case 1 ,

$$
G_{r-1} \perp \hat{G}_{r-1}, \quad\left\|G_{r-1}\right\| \leq 1, \quad\left\|\hat{G}_{r-1}\right\| \leq 1, \quad \text { and } \quad \operatorname{rank} G_{r-1}+\operatorname{rank} \hat{G}_{r-1} \leq k .
$$

Therefore, $\left\|G_{r}\right\| \leq 1$ and $\operatorname{rank} G_{r} \leq k$. Since

$$
\left\|G_{r}+\alpha \hat{G}_{r}\right\|_{(k)}=k \quad \text { and } \quad\left\|2 G_{r}+\beta \hat{G}_{r}\right\|_{(k)}=\left\|G_{r}\right\|_{(k)}+\left\|G_{r}+\beta \hat{G}_{r}\right\|_{(k)},
$$

for any complex unit $\alpha$, by Lemma 2.4, we obtain $G_{r} \perp \hat{G}_{r}$. Moreover, $G_{r}$ has singular values 0 and 1 only. Similarly, one can conclude that $\hat{G}_{r}$ has singular values 0 and 1 only. Now assume that the claim holds for some $r>\log _{2} k$. We will show that the claim also holds for $r+1$. By induction assumption and the fact that $G_{r+1}=G_{r}+\hat{G}_{r}$, we conclude that $G_{r+1}$ has singular values 0 and 1 only. The same conclusion holds for $\hat{G}_{r+1}$. By Lemma 2.5 and the fact that $\left\|G_{r+1}+\alpha \hat{G}_{r+1}\right\|=k$ for all complex unit $\alpha$, we get $G_{r+1} \perp \hat{G}_{r+1}$. Therefore the claim holds.

Combining the above two cases, we see that (3.4) holds and hence the statement (3.3) follows. Therefore, the claim (3.2) holds for all $k$. Without loss of generality, we may assume $U=V=I_{N}$ in (3.2). Following a similar argument as in Theorems 2.1 and 2.6, one can conclude that

$$
\phi\left(E_{j_{1} j_{1}} \otimes \cdots \otimes E_{j_{m-1} j_{m-1}} \otimes B\right)=E_{j_{1} j_{1}} \otimes \cdots \otimes E_{j_{m-1} j_{m-1}} \otimes \varphi_{j_{1}, \ldots, j_{m-1}}(B)
$$

for all $1 \leq j_{i} \leq n_{i}$ with $1 \leq i \leq m-1$ and $B \in M_{n_{m}}$, where $\varphi_{j_{1}, \ldots, j_{m-1}}$ can be assumed to be either the identity map or the transposition map. Following the same argument, we can further conclude that for any $X=X_{1} \otimes \cdots \otimes X_{m-1}$ with $X_{i} \in M_{n_{i}}$ being unitary for $1 \leq i \leq m-1$, there are unitary $U_{X}$ and $V_{X}$ such that

$$
\phi\left(\left(\bigotimes_{i=1}^{m-1} X_{i} E_{j_{i} j_{i}} X_{i}^{*}\right) \otimes B\right)=U_{X}\left(\left(\bigotimes_{i=1}^{m-1} X_{i} E_{j_{i} j_{i}} X_{i}^{*}\right) \otimes \varphi_{j_{1}, \ldots, j_{m-1}, X}(B)\right) V_{X}
$$

for all $1 \leq j_{i} \leq n_{i}$ with $1 \leq i \leq m-1$ and $B \in M_{n_{m}}$, where $\varphi_{j_{1}, \ldots, j_{m-1}, X}$ can be assumed to be either the identity map or the transposition map, depending on $j_{1}, \ldots, j_{m-1}$ and $X$. By the fact that $\phi\left(I_{N}\right)=I_{N}$, we have $V_{X}^{*}=U_{X}$.

Again, considering all symmetric $S \in M_{n_{m}}$ as in the proof of Theorem 2.1, we can show that there exists $W_{X} \in M_{n_{1} \cdots n_{m-1}}$ such that

$$
\phi\left(\left(\bigotimes_{i=1}^{m-1} X_{i} E_{j_{i} j_{i}} X_{i}^{*}\right) \otimes B\right)=W_{X}\left(\bigotimes_{i=1}^{m-1} X_{i} E_{j_{i} j_{i}} X_{i}^{*}\right) W_{X}^{*} \otimes \varphi_{j_{1}, \ldots, j_{m-1}, X}(B)
$$

for all $1 \leq j_{i} \leq n_{i}$ with $1 \leq i \leq m-1$ and $B \in M_{n_{m}}$. Consider the linear map (known as the partial trace function in quantum information science context) on $M_{N}$ defined by $X \otimes Y \mapsto(\operatorname{tr} X) Y$ for $X \in M_{n_{1} \cdots n_{m-1}}$ and $Y \in M_{n_{m}}$ and apply to the above equation, we see that every choice of $\bigotimes_{j=1}^{m-1} X_{i} E_{j_{i} j_{i}} X_{i}^{*}$ gives rise to a linear map $\varphi_{j_{1}, \ldots, j_{m}, X}$, which is either the identity map or the transposition map. Evidently, the map

$$
\bigotimes_{j=1}^{m-1} X_{i} E_{j_{i} j_{i}} X_{i}^{*} \mapsto \varphi_{j_{1}, \ldots, j_{m}, X}
$$


is linear and hence continuous; the set

$$
\begin{aligned}
&\left\{\bigotimes_{j=1}^{m-1} X_{i} E_{j_{i} j_{i}} X_{i}^{*}: 1 \leq j_{i} \leq n_{i} \text { and } X_{i}^{*} X_{i}=I_{n_{i}}, \text { for } i=1, \ldots, m-1\right\} \\
&=\left\{x_{1} x_{1}^{*} \otimes \cdots \otimes x_{m-1} x_{m-1}^{*}: x_{i} \in \mathbb{C}^{n_{i}} \text { with } 1 \leq i \leq m-1\right\}
\end{aligned}
$$

is connected. Thus, all the maps $\varphi_{j_{1}, \ldots, j_{m-1}, X}$ have to be the same. Assume that this common map is $\varphi_{m}$, which is either the identity map or the transposition map. By linearity, one can conclude that for any $A_{1} \otimes \cdots \otimes A_{m} \in M_{n_{1}} \otimes \cdots \otimes M_{n_{m}}$, we have

$$
\phi\left(A_{1} \otimes \cdots \otimes A_{m}\right)=\psi\left(A_{1} \otimes \cdots \otimes A_{m-1}\right) \otimes \varphi_{m}\left(A_{m}\right)
$$

for some $\psi\left(A_{1} \otimes \cdots \otimes A_{m-1}\right) \in M_{n_{1} \cdots n_{m-1}}$. Note that $\psi: M_{n_{1} \cdots n_{m-1}} \rightarrow M_{n_{1} \cdots n_{m-1}}$ preserves the Ky Fan $k$-norm of all matrices in $M_{n_{1}} \otimes \cdots \otimes M_{n_{m-1}}$. By the induction hypothesis, we know there exist unitary $\tilde{U}, \tilde{V}$ such that

$$
\psi\left(A_{1} \otimes \cdots \otimes A_{m-1}\right)=\tilde{U}\left(\varphi_{1}\left(A_{1}\right) \otimes \cdots \otimes \varphi_{m-1}\left(A_{m-1}\right)\right) \tilde{V}
$$

with each $\varphi_{j}$ being either the identity map or the transposition map. Hence, $\phi$ has the desired form and the proof is completed.

Using a similar argument and applying Lemma 2.7, we can extend Theorem 2.9 to multipartite systems as follows.

TheOREM 3.2. Let $1 \leq p<\infty$ and $p \neq 2$ and $\phi: M_{n_{1} \cdots n_{m}} \rightarrow M_{n_{1} \cdots n_{m}}$ be a linear map. The following are equivalent.

(a) $\left\|\phi\left(A_{1} \otimes \cdots \otimes A_{m}\right)\right\|_{p}=\left\|A_{1} \otimes \cdots \otimes A_{m}\right\|_{p}$ for all $A_{i} \in M_{n_{i}}, i=1, \ldots, m$.

(b) There are unitary matrices $U, V \in M_{n_{1} \cdots n_{m}}$ such that

$$
\phi\left(A_{1} \otimes \cdots \otimes A_{m}\right)=U\left(\varphi_{1}\left(A_{1}\right) \otimes \cdots \otimes \varphi_{m}\left(A_{m}\right)\right) V, \quad \text { for all } \quad A_{i} \in M_{n_{i}}, i=1, \ldots, m,
$$

where $\varphi_{s}$ is the identity map or the transposition map $X \mapsto X^{t}$ for $s=1, \ldots, m$.

\section{Acknowledgment}

This research was supported by a Hong Kong GRC grant PolyU 502411 with Sze as the PI. The grant also supported the post-doctoral fellowship of Huang and the visit of Fošner to the Hong Kong Polytechnic University in the spring of 2011. She gratefully acknowledged the support and kind hospitality from the host university. Li was supported by a GRC grant and a USA NSF grant; this research was done when he was a visiting professor of the University of Hong Kong in the spring of 2012; furthermore, he is an honorary professor of Taiyuan University of Technology (100 Talent Program scholar), and an honorary professor of the Shanghai University. 


\section{REFERENCES}

[1] J. Arazy, The isometries of $C_{p}$, Israel J. Math. 22 (1975), 247-256.

[2] N. Bourbaki, Elements of mathematics, Algebra I, Springer-Verlag, New York, 1989.

[3] J.T. Chan, C.K. Li, and N.S. Sze, Isometries for unitarily invariant norms, Linear Algebra Appl. 399 (2005), 53-70.

[4] K. Chen and L.A. Wu, A matrix realignment method for recognizing entanglement, Quantum Inf. Comput. 3 (2003), 193.

[5] A. Fošner, Z. Huang, C.K. Li, and N.S. Sze, Linear preservers and quantum information science, Linear and Multilinear Algebra, to appear. (Preprint available online http://dx.doi.org/10.1080/03081087.2012.740029)

[6] S. Friedland, C.K. Li, Y.T. Poon, and N.S. Sze, The automorphism group of separable states in quantum information theory, J. Math. Phys., 52 (2011), 042203.

[7] R. Grone and M. Marcus, Isometries of matrix algebras, J. Algebra 47 (1977), 180-189.

[8] R.A. Horn and C.R. Johnson, Matrix Analysis, Cambridge University Press, Cambridge, 1985.

[9] A.K. Jain, Fundamentals of Digital Image Processing, Prentice Hall, New Jersey, 1989.

[10] N. Johnston, Characterizing Operations Preserving Separability Measures via Linear Preserver Problems, Linear and Multilinear Algebra 59 (2011), 1171-1187.

[11] N. Johnston and D.W. Kribs, A family of norms with applications in quantum information theory, J. Math. Phys. 51 (2010), 082202.

[12] C.K. Li, Matrices with some extremal properties, Linear Algebra Appl. 101 (1988), 255-267.

[13] C.K. Li and S. Pierce, Linear preserver problems, Amer. Math. Monthly 108 (2001), 591-605.

[14] C.K. Li, Y.T. Poon, and N.S. Sze, Linear preservers of tensor product of unitary orbits, and product numerical range, Linear Algebra Appl., to appear. (Preprint available online http://dx.doi.org/10.1016/j.laa.2011.07.039)

[15] C.K. Li, Y.T. Poon, and N.S. Sze, Isometries for Ky Fan norms between matrix spaces, Proc. Amer. Math. Soc. 133 (2005) 369-377.

[16] C.K. Li, P. Šemrl, and A.R. Sourour, Isometries for Ky-Fan norms on block triangular matrix algebras, Archiv Math. 81 (2003), 175-181.

[17] C.A. McCarthy, $C_{p}$, Israel J. Math. 5 (1967), 249-271.

[18] S. Mac Lane and B. Birkhoff, Algebra, AMS Chelsea, Providence, 1999.

[19] M.A. Nielsen and I.L. Chuang, Quantum Computation and Quantum Information, Cambridge University Press, 2000.

[20] O. Rudolph, Further results on the cross norm criterion for separability, Quantum Inf. Process. 4 (2005), 219.

[21] S. Willi-Hans and H. Yorick, Matrix Calculus And Kronecker Product: A Practical Approach to Linear and Multilinear Algebra, 2nd Edition, World Scientific, Singapore, 2011. 